\title{
Flight Test Evaluation of the ATD-1 Interval Management Application
}

\author{
Kurt A. Swieringa ${ }^{1}$, Sara R. Wilson ${ }^{2}$, Brian T. Baxley ${ }^{3}$, Roy D. Roper ${ }^{4}$ \\ NASA Langley Research Center, Hampton, VA, 23681 \\ Terence S. Abbott ${ }^{5}$ \\ Science Applications International Corporation \\ Ian Levitt ${ }^{6}$ \\ Federal Aviation Administration, Atlantic City, NJ, 08405 \\ and \\ Julien $\operatorname{Scharl}^{7}$ \\ Boeing Company, Everett, WA, 98204
}

\begin{abstract}
Interval Management (IM) is a concept designed to be used by air traffic controllers and flight crews to more efficiently and precisely manage inter-aircraft spacing. Both government and industry have been working together to develop the IM concept and standards for both ground automation and supporting avionics. NASA contracted with Boeing, Honeywell, and United Airlines to build and flight test an avionics prototype based on NASA's spacing algorithm and conduct a flight test. The flight test investigated four different types of IM operations over the course of nineteen days, and included en route, arrival, and final approach phases of flight. This paper examines the spacing accuracy achieved during the flight test and the rate of speed commands provided to the flight crew. Many of the time-based IM operations met or exceeded the operational design goals set out in the standards for the maintain operations and a subset of the achieve operations. Those operations which did not meet the goals were due to issues that are identified and will be further analyzed.
\end{abstract}

\section{Nomenclature}

$\begin{array}{ll}A B P & =\text { Achieve-By Point } \\ A D S-B & =\text { Automatic Dependent Surveillance }- \text { Broadcast } \\ A S G & =\text { Assigned Spacing Goal } \\ A S T A R & =\text { Airborne Spacing for Terminal Arrival Routes spacing algorithm } \\ A T D-1 & =\text { First Air Traffic Management Technology Demonstration } \\ C M S & =\text { Controller Managed Spacing } \\ E F B & =\text { Electronic Flight Bag } \\ F A A & =\text { Federal Aviation Administration } \\ F A F & =\text { Final Approach Fix } \\ F I M & =\text { Flight deck Interval Management } \\ F M S & =\text { Flight Management System } \\ G I M-S & =\text { Ground-based Interval Management }- \text { Spacing } \\ I M & =\text { Interval Management }\end{array}$

${ }^{1}$ Research Engineer, Crew Systems and Aviation Operations Branch, NASA LaRC, MS 152, AIAA Member.

${ }^{2}$ Research Engineer, Systems Engineering and Engineering Methods Branch, NASA LaRC, MS 290.

${ }^{3}$ Senior Research Engineer, Crew Systems and Aviation Operations Branch, NASA LaRC, MS 152, AIAA Senior Member.

${ }^{4}$ Research Aerospace Engineer, Crew Systems and Aviation Operations Branch, NASA LaRC, MS 152.

${ }^{5}$ Senior Research Engineer, Science Applications International Corporation.

${ }^{6}$ Mathematician, Federal Aviation Administration William J Hughes Technical Center, Atlantic City, NJ 08405.

${ }^{7}$ Associate Technical Fellow, Boeing Company, Modeling and Simulation, AIAA Member.

American Institute of Aeronautics and Astronautics 


$\begin{array}{ll}K B F I & =\text { Boeing Field } / \text { King County International Airport } \\ K M W H & =\text { Grant County International Airport } \\ K S E A & =\text { Seattle-Tacoma International Airport } \\ M O P S & =\text { Minimum Operational Performance Standards } \\ \text { NAS } & =\text { National Airspace System } \\ N A S A & =\text { National Aeronautics and Space Administration } \\ N O A A & =\text { National Oceanic and Atmospheric Administration } \\ P T P & =\text { Planned Termination Point } \\ R N A V & =\text { Area Navigation } \\ R N P & =\text { Required Navigation Performance } \\ \text { SD } & =\text { Standard Deviation } \\ S T A R & =\text { Standard Terminal Arrival Route } \\ T M A-T M & =\text { Management Advisor with Terminal Metering } \\ T R A C O N & =\text { Terminal Radar Approach Control } \\ T S A S & =\text { Terminal Sequencing and } \\ Z S E & =\text { Seattle Air Route Traffic Control Centers }\end{array}$

\section{Introduction}

S the number of commercial aircraft operations increases, government and industry are developing new concepts and technologies to improve the capacity and efficiency of the National Airspace System (NAS). Interval Management (IM) is a NextGen concept that is enabled by the integration of ground and flight-deck capabilities and procedures designed to be used by air traffic controllers and flight crews to more efficiently and precisely manage inter-aircraft spacing. One of the benefits of IM is derived from the enhanced delivery accuracy that it offers. If aircraft can achieve more consistent inter-arrival spacing at flow restricted points, it may be possible to reduce the spacing buffer used by air traffic controllers to prevent separation violations, resulting in increased throughput. Research into spacing applications dates back to the 1970's and early 1980 's $^{1-3}$ and across several different organizations. A comprehensive history of IM research is described in reference 4.

The goal of the National Aeronautics and Space Administration's (NASA's) first Air Traffic Management Technology Demonstration (ATD-1) is to demonstrate technology that enables aircraft to use speed control to achieve an integrated arrival schedule, enabling the use of performance-based navigation procedures during peak traffic demand. In order to accomplish this goal, NASA developed and tested prototypes of three different technologies whose integrated use improves the efficiency of arrivals into busy airports and supports the implementation of NextGen. The first two technologies were the Traffic Management Advisor with Terminal Metering (TMA-TM) and Controller Managed Spacing (CMS), which provide terminal air traffic controllers with a deconflicted arrival schedule and decision support tools to help them manage to the schedule. ${ }^{5-9}$ Both TMA-TM and CMS were evaluated at the Federal Aviation Administration's (FAA's) William J. Hughes Technical Center in 2015 and transferred to the FAA, forming the basis for the ongoing acquisition of Terminal Sequencing and Spacing (TSAS). ${ }^{10}$ The third technology was a prototype of a Flight-deck based Interval Management (FIM) application that included NASA's Airborne Spacing for Terminal Arrival Routes (ASTAR) spacing algorithm. FIM is the ADS-B In application that enables the flight-deck component of IM. FIM avionics provide pilots with speeds that they can fly to achieve or maintain a precise spacing interval behind another aircraft, referred to as the Target aircraft.

As part of the agency's mission to deliver NextGen benefits, the Federal Aviation Administration (FAA) has been establishing operational and technical requirements for IM. This includes integrating ground metering tools, such as TSAS and Ground-based Interval Management - Spacing (GIM-S), with airborne tools, such as FIM, into cohesive arrival and approach operations. Part of this development involves working with industry to develop Safety and Performance Requirements (SPR) for the IM Operations and Minimum Operational Performance Standards (MOPS) for the FIM avionics. ${ }^{11,12}$ The first version of the FIM MOPS was published in September 2015 and is currently under revision to support additional capabilities and greater aircraft integration. These industry standards complement ongoing acquisition efforts by the FAA to develop and deploy ground-based metering systems into the NAS.

ATD-1's primary contribution to the IM research was the development and demonstration of prototype FIM and TSAS systems and an examination of the integration of FIM with TSAS. Several simulations were conducted in preparation for the ATD-1 flight demonstration, ${ }^{13-15}$ and a flight demonstration of NASA's ASTAR algorithm was conducted onboard Boeing's ecoDemonstrator aircraft. ${ }^{16}$ The final human-in-the-loop simulation conducted by NASA under ATD-1 took place in August 2015. ${ }^{17-19}$

American Institute of Aeronautics and Astronautics 
This work culminated in a NASA contract with Boeing, Honeywell, and United Airlines to develop a FIM avionics prototype based on the ASTAR spacing algorithm and conduct a flight test to evaluate the airborne performance of that prototype..$^{20}$ The FIM avionics created for the flight test extended ASTAR to include most of the FIM MOPS functionality, supporting spacing operations on final approach, during cruise, and using both time- and distance-based spacing. This paper describes the results and conclusions of the flight test. Particular attention is given to the spacing accuracy of the FIM avionics and the rate of commanded speeds that were provided to the pilots. A separate paper is available that provides additional results focusing on the flight crew responses given in the end-of-run and end-of-day surveys. ${ }^{21}$

\section{IM Operations}

Interval Management (IM) consists of a set of ground and flight-deck capabilities and procedures that are used by air traffic controllers and flight crews to more efficiently and precisely manage inter-aircraft spacing. In the operational and technical standards, IM operations are defined in terms of the IM clearances that are provided by the controller. ${ }^{11}$ These clearances include an Assigned Spacing Goal (ASG), which is a time or distance interval between the IM and Target aircraft, assigned by the controller and usually derived from metering information. The ASG can either be achieved at the Achieve-by Point (ABP) or maintained until the Planned Termination Point (PTP). IM Operations are defined for the en route, arrival, and approach phases of flight, using both time-based and distance-based ASGs.

IM operations can be composed of a maintain stage and an achieve stage. The goal of the maintain stage is to maintain the ASG until the PTP. The maintain stage is designed to use state information from the Target aircraft to determine the spacing error; therefore, a state-based control law that is restricted to in-trail operations is used. The goal of the achieve stage is to achieve the ASG when crossing the ABP. The achieve stage is designed to use trajectory information from the IM and Target aircraft to determine the spacing error; therefore, a trajectory-based control law that can support merging routes is used.

The achieve and maintain stages are procedurally combined into distinct IM clearances. The four clearances exercised in the ATD-1 flight test were Maintain, Capture, Cross, and Final Approach Spacing.

- The Maintain clearance is used when the IM and Target aircraft are following a common route and the controller wants the IM aircraft to maintain the current in-trail spacing, as determined by the FIM avionics. The algorithm determines speeds that will continuously maintain the in-trail spacing within 10 seconds until the operation terminates. This clearance is intended for tactical use, when aircraft already have an in-trail spacing that the controller wants. Within this flight test, the Maintain clearance was used during en route and arrival operations.

- The Capture clearance is used when the IM and Target aircraft are on a common route and the controller wants the IM aircraft to achieve the ASG quickly and then maintain it until termination. The algorithm determines speeds that will immediately correct the initial spacing error, and then maintain the in-trail spacing within 10 seconds of the ASG until the operation terminates. This clearance is intended for use when the spacing between the IM and Target aircraft are close to the spacing interval that the controller or schedule needs. Within this flight test, the Capture clearance was used during en route and arrival operations.

- The Cross clearance is used when the controller wants the IM aircraft to achieve the ASG at the ABP, and then maintain the ASG until termination. The achieve stage is used to correct the initial spacing error by the $\mathrm{ABP}$, and then transitions to the maintain stage until termination. This clearance is meant for strategic use, with the ASG derived from metering information. Within this flight test, the Cross clearance was also used during both en route and arrival operations.

- The Final Approach Spacing clearance is used when the final controller wants to use IM to control the rate of compression or overtake with a preceding arrival on final. This clearance is meant for tactical use, and the controller determines an ASG that improves the precision of the interval between successive arrivals. Within this flight test, the Final Approach Spacing clearance was given to one aircraft either established on final or on a vector to intercept the final approach course.

All clearances can be used with a time-based ASG, and this is the expected norm. Distance-based ASGs can be used, but not during the maintain stage when aircraft are descending or decelerating. In the ATD-1 flight test, distancebased ASGs were only used during the en route cruise operations and the Cross clearances which terminated at the ABP.

American Institute of Aeronautics and Astronautics 


\section{Flight Test Description}

\section{A. Scenario Design}

\section{Flight Test Operations}

The ATD-1 flight test took place in Seattle en route (ZSE) and Moses Lake terminal (KMWH) airspace. Three aircraft departed from Boeing Field (KBFI) and Seattle-Tacoma International Airport (KSEA). While en route, two of the aircraft equipped with the FIM avionics conduced either Maintain or Capture operations. After the en route operation was completed, the aircraft conducted a series of arrival operations into Grant County International Airport (KMWH). In order to condition the aircraft for these operations, the flight test director coordinated with flight crews and ZSE controllers. On select days, after the approach scenarios were completed Moses Lake controllers provided vectors to two of the aircraft to set up Final Approach Spacing operations.

\section{Airspace Design}

The two Standard Terminal Arrival Routes (STARs) designed for the flight test were named the SUBDY1 arrival and UPBOB1 arrival (red lines in Figure 1). These STARS are connected to the published Area Navigation (RNAV) and Required Navigation Performance (RNP) Z approaches to runway $32 \mathrm{R}$, creating a continuous route to the runway. Throughout the flight test both the Ownship and Target aircraft were assigned STARs and transitions to create different merging conditions. The SUBDY 1 arrival has two transitions that create a medium altitude merge condition and both the SUBDY1 and UPBOB1 arrivals merge at the Final Approach Fix (FAF),

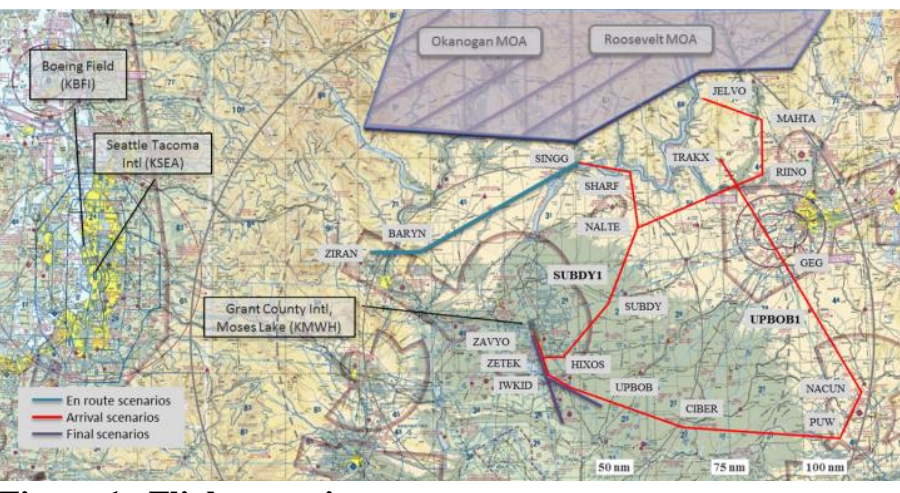

Figure 1. Flight test airspace. creating a low altitude merge condition.

Hold points and IM initiation points were selected to prevent the aircraft from entering special use airspace to the north and from crossing certain sector boundaries, reducing the amount of coordination with air traffic control required to conduct the flight test. The planned IM initiation points for arrival operations were approximately $90 \mathrm{nmi}$ from the runway. However, the actual length of the arrival operations ranged from approximately 40 to $130 \mathrm{nmi}^{*}$

Routes were also defined to support en route and Final Approach Spacing operations. A cruise route defined by the ZIRAN, BARYN, and SINGG waypoints (purple line in Figure 1) was typically flown at FL350. In order to support Final Approach Spacing operations, controllers vectored aircraft onto either an extended final approach segment or straight segment that proceeded direct-to the final approach segment (purple lines in Figure 1).

\section{Lead Aircraft Delayed Speed Profiles}

When time-based metering is used, traffic flow management systems, such as Time Based Flow Management with TSAS enhancements, will allocate delay to aircraft in order to deconflict them at a series of meter points. Since there is no current method of communicating trajectories between traffic flow management systems and aircraft conducting IM operations, the FIM avionics use the published speeds instead of the delayed speeds to estimate the times of arrival for the Ownship and Target aircraft. This presents an unknown source of error that is expected to affect the performance of the Cross operations, and may also affect the performance of the Maintain and Capture operations.

Even though TSAS was not used in this flight test, there was a desire to emulate the unknown delay that an aircraft would have if controlled by air traffic controllers utilizing TSAS and time-based metering. Figure 2 shows three different delayed speed profiles that were created and flown by the aircraft leading the string of IM aircraft, referred to as the lead aircraft. The No Delay speed profiles (black lines) followed the nominal published speeds. The Moderate Delay speed profiles (blue lines) had a moderate amount of delay in the TRACON (approximately 20 sec) and no delay in the Center. The High Delay speed profiles (red lines) had maximum delay in the TRACON (approximately $40 \mathrm{sec}$ ) and moderate delay in the Center (approximately $25 \mathrm{sec}$ ). Figure 2 also shows the upper and lower bounds on

\footnotetext{
* There are two main factors that impacted the length of IM operations. First, the flight crew of the IM aircraft had to coordinate with the flight test director if the aircraft setup did not allow for the desired test condition. This coordination took time and delayed the start of the IM operation. Secondly, the data was filtered after the flight test to remove the impact of software anomalies, reducing the length of particular operations.
} 
the speeds that the FIM avionics can command (grey dotted lines) and a reference slow speed profile (solid grey line) that emulates both the slowest speed that TSAS can use when allocating delay and the slowest speed the aircraft are expected to fly in the Center airspace.

The process of creating the speed profiles began with generating a reference slow speed profile. In the TRACON, the slow speed profile was determined by subtracting 20 knots from the nominal speed, which was similar to adaptations used in previous ATD-1 simulations. ${ }^{14,15,17}$ In the Center airspace, it was assumed that commercial aircraft fly a minimum speed of 250 knots to absorb delay after descending below their Mach/CAS transition altitude. Based on how traffic flow management systems allocate delay, delay was first allocated to the TRACON and then to the Center.

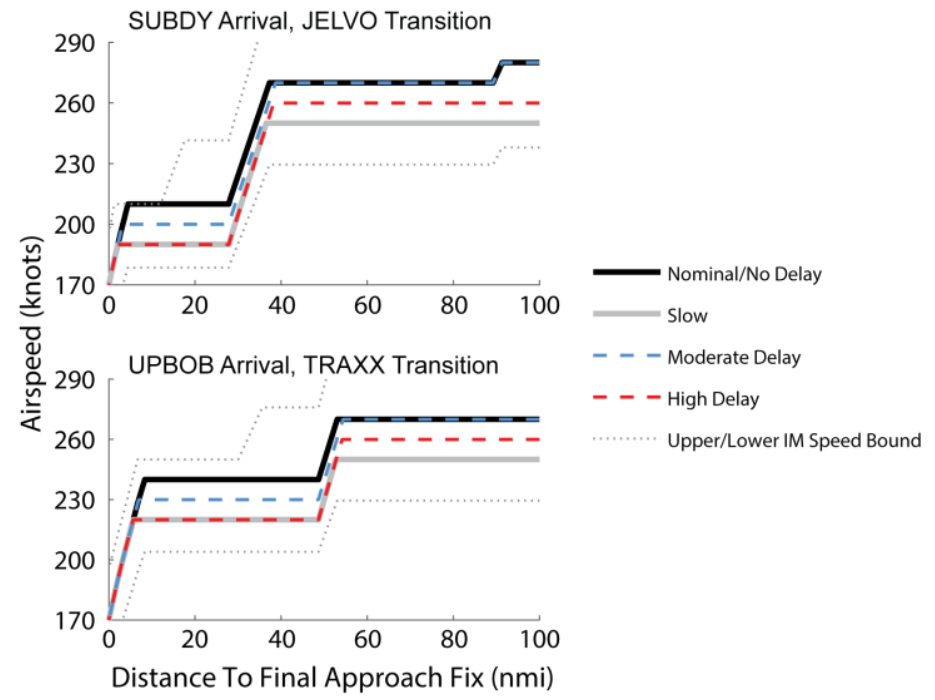

Figure 2. The delayed speed profiles flown by the lead aircraft.

\section{Wind Forecast}

Both the Flight Management System (FMS) and the FIM avionics used a single wind forecast determined at the beginning of each flight test day from the National Oceanic and Atmospheric Administration (NOAA) Aviation Weather Service. Since descent winds were not available for Grant County International Airport, they were averaged between Yakima and Spokane International Airport. For the FIM avionics, FL340 was the altitude used for the en route forecast, and FL240, FL180, 12,000 feet, 6000 feet, and the surface were the altitudes used for the descent forecast. To standardize data entry between the two FMS types and to align with the FIM avionics, FL340 was the altitude used for the FMS en route forecast, and FL180, 12000 feet, and 6000 feet were the altitudes used for the FMS descent forecast.

\section{B. Independent Variables}

This flight test investigated IM operations during three phases of flight: en route, arrival, and final approach. The original test plan included 124 data points (two replicates of 62 unique scenarios); ${ }^{22}$ however, tactical decisions made during the flight test resulted in missing, invalid, and additional data points for particular scenarios. In the end, there were 144 successful data points collected: 125 from arrival operations, 11 from en route operations, and 8 from final approach spacing operations. ${ }^{\dagger}$

In order to minimize the impact of schedule constraints and potential weather, the run order was designed to ensure the highest priority scenarios were flown, while also minimizing the impact of systematic bias and order effects on the results. The replicates of each scenario were assigned a priority level and the run order of the scenarios was randomized within each priority level.

\section{Arrival Scenarios}

The arrival scenarios consisted of IM operations that started at an altitude of either FL350 or FL230 and terminated at the FAF. The arrival scenarios flown during the flight test were designed to evaluate five independent variables: the IM clearance type, whether the ASG was distance- or time-based, lead aircraft delay, initial spacing error, and whether the ABP location was at a medium altitude merge point or the final approach fix (Table 1). Not all combinations of variables were able to be investigated. For example, the location of the ABP only applies to the Cross clearance, and no distance-based clearances included a maintain stage during the descent. Since previous ATD-1 research was focused on the use of IM in a metered environment, a strong emphasis was placed on the time-based arrival operations. Of the 125 arrival data points, 118 were time-based and 7 were distance-based.

The initial spacing error was varied across the test, as depicted in Figure 3. The planned spacing error for the Capture and Cross clearances ranged from 60 seconds early to 60 seconds late for time-based operations and from 1

\footnotetext{
${ }^{\dagger}$ There were an additional 13 successfully data points collected at the end of the flight test to examine modifications to the speed constraints on the arrival and approach procedures; however, those data points are not discussed in this paper.
}

American Institute of Aeronautics and Astronautics 
Table 1: Test matrix for arrival scenarios

\begin{tabular}{|c|c|c|c|}
\hline $\begin{array}{c}\text { IM Clearance } \\
\text { Type }\end{array}$ & $\begin{array}{c}\text { Time- or } \\
\text { Distance-based }\end{array}$ & $\begin{array}{c}\text { Lead Aircraft } \\
\text { Delay }\end{array}$ & $\mathbf{N}$ \\
\hline \multirow{3}{*}{ Maintain } & \multirow{3}{*}{ Time } & None & 6 \\
\hline & & Medium & 8 \\
\hline & & High & 4 \\
\hline \multirow{3}{*}{ Capture } & \multirow{3}{*}{ Time } & None & 17 \\
\hline & & Medium & 7 \\
\hline & & High & 8 \\
\hline \multirow{3}{*}{ Cross-Merge ${ }^{*}$} & \multirow{3}{*}{ Time } & None & 10 \\
\hline & & Medium & 9 \\
\hline & & High & 8 \\
\hline \multirow{6}{*}{ Cross-FAF ${ }^{* *}$} & \multirow{3}{*}{ Time } & None & 14 \\
\hline & & Medium & 11 \\
\hline & & High & 16 \\
\hline & \multirow{3}{*}{ Distance } & None & 4 \\
\hline & & Medium & 1 \\
\hline & & High & 2 \\
\hline
\end{tabular}

* Achieve-by point at a medium-altitude merge point

** Achieve-by point at the FAF

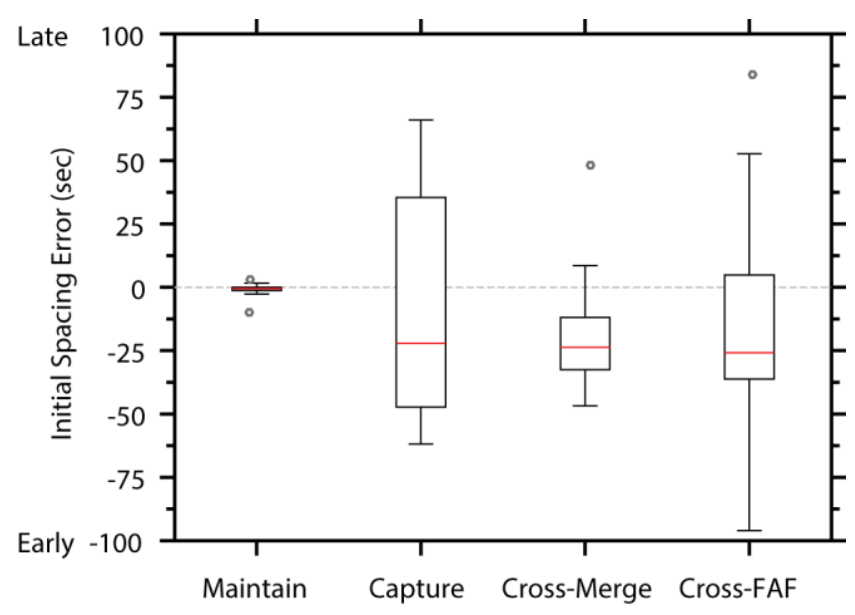

Figure 3. Initial spacing error for time-based arrivals.

nmi early to $2 \mathrm{nmi}$ late for distance-based operations. All of the initial spacing errors for the Maintain operation were close to zero because its objective is to maintain the initial spacing interval. The initial spacing error was challenging to control for and the flight test director often had to accept initial spacing errors that were different than planned. Some of these initial spacing errors exceeded what is expected operationally, resulting in stressful test conditions.

\section{En Route Scenarios}

The four planned high-altitude en route scenarios flown during the flight test were designed to evaluate three independent variables: IM Clearance Type, time- or distance-based ASG, and initial spacing error. The two clearance types that were investigated were the Maintain and Capture clearances. Of the eleven en route operations conducted, six were time-based and five were distance-based. The initial spacing error for the Capture operations ranged from 23 seconds early to 20 seconds early for time-based operations and from 3.8 nmi early to 2.6 nmi late for distance-based operations.

\section{Final Approach Spacing Scenarios}

The six planned final approach scenarios flown during the flight test were designed to evaluate two independent variables: merge geometry and time- or distance-based ASG. For all of the final approach scenarios both the ABP and the PTP were located at the default location specified in the MOPS, $6.25 \mathrm{nmi}$ from the runway threshold. Of the eight Final Approach Spacing operations flown, five were time-based operations with initial spacing errors that ranged from 23 seconds early to 29 seconds late, and three were distance-based operations with initial spacing errors that ranged from $1.5 \mathrm{nmi}$ early to $0.1 \mathrm{nmi}$ early. Unlike the arrival and en route scenarios, only one IM aircraft was used during the Final Approach Spacing operations.

\section{Flight Test Aircraft}

Three aircraft participated in the flight test (Figure 4). A Dassault Falcon-900, provided by Honeywell, was used as the lead aircraft for a majority of the flight test. A Boeing 757-200 provided by Honeywell and a Boeing 737-900 provided by United Airlines were equipped with ADS-B In and the FIM avionics prototype. All three aircraft were equipped with ADS-B Out, Global Navigation Satellite System, were Area Navigation capable, and were authorized to fly the published Required Navigation Performance Authorization Required approaches into the Grant County International Airport.

\section{Typical Flight Test Day}

During future operations, aircraft equipped with FIM avionics will be preconditioned using metering tools such as GIM-S and TSAS. Since these tools were not available during the flight test, a flight test director, the flight test pilots and air traffic controllers worked together to position the aircraft for each test condition.

On a typical flight test day, the United Boeing-737 departed from KSEA and the Honeywell Boeing-757 and Falcon-900 departed from KBFI. The tower facilities at KBFI and KSEA coordinated with the Seattle TRACON to determine departures times that resulted in a spacing interval of approximately $20 \mathrm{nmi}$ when the aircraft reached their cruise altitude of FL350. When the aircraft equipped with FIM avionics reached ZIRAN, they initiated an en route IM 


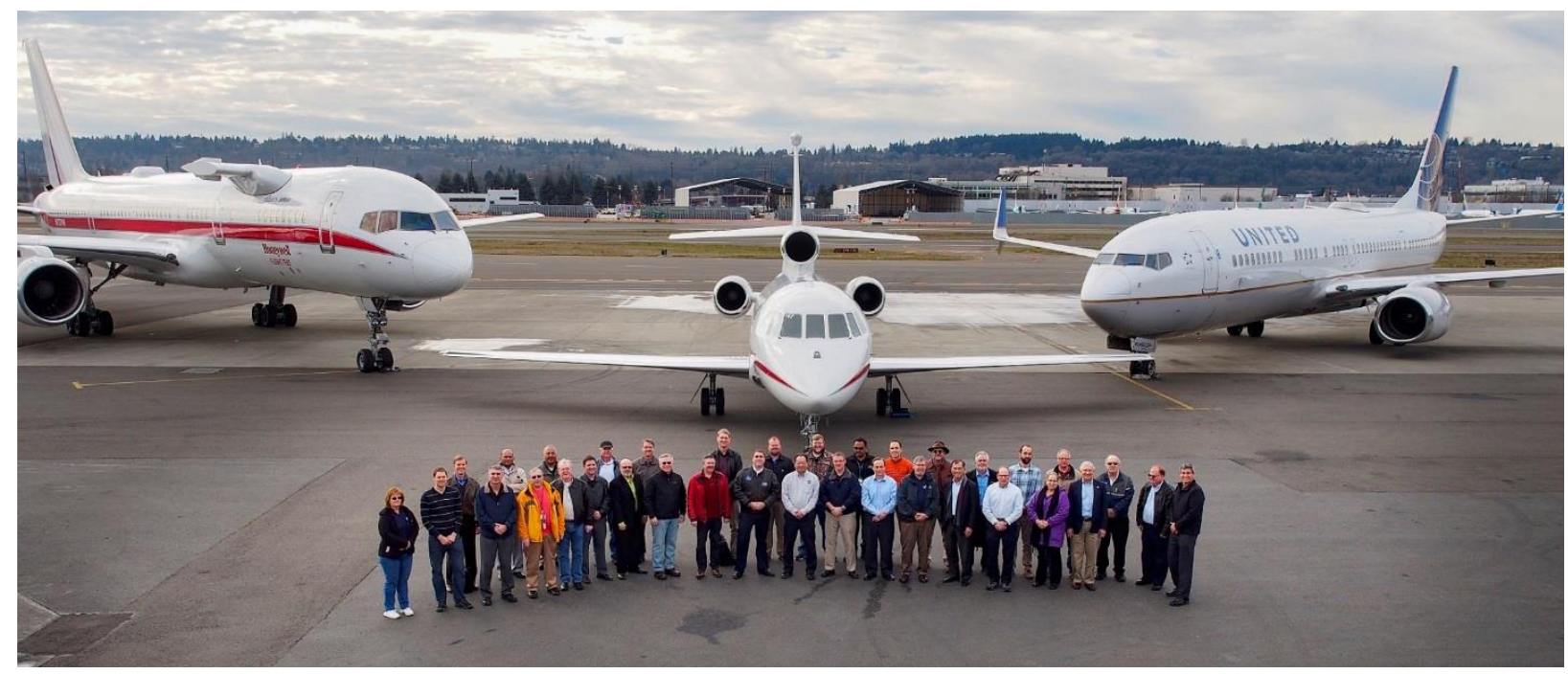

Figure 4. Aircraft used in ATD-1 flight test.

operation that terminated at SINGG. After the aircraft crossed the PTP the flight crews entered information into the FIM avionics for the first arrival scenario, either the SUBDY1 or UPBOB1 arrival that connected to an approach to runway $32 \mathrm{R}$.

For the operations that terminated at the FAF, the aircraft continued descending to their decision altitude, conducted a missed approach, and then proceeded to the initial points for the next operation. During climb out, each flight crew used their FMS to estimate their flight time to either the FAF or a common merge point and provided that time to the flight test director, who issued each flight crew a scheduled time of arrival to that waypoint. The flight crews independently coordinated with air traffic control to determine a set of maneuvers that enabled them to arrive at the initiation point at the desired time. When the aircraft reached their initiation point the flight crews used the FIM avionics to determine the current spacing interval, which was used to calculate an ASG that yielded the desired initial spacing error. If the calculated ASG was outside the bounds defined for this flight test (e.g., 150 to 210 seconds), the flight crew coordinated with the flight test director, who determined an alternative ASG. This process was repeated for each of the planned arrival scenarios.

After the arrival operations were completed the Falcon-900 returned to KBFI. If Final Approach Spacing operations were planned, Moses Lake TRACON controllers provided the Boeing- 757 and the Boeing- 737 with vectors to set up the Final Approach Spacing operations. Similarly to the en route and arrival scenarios, the pilots either modified their ASG to obtain the desired initial spacing error or coordinated with the flight test director to select an alternate ASG.

\section{E. FIM Avionics}

\section{FIM Prototype}

Figure 5 shows the prototype electronic flight bag (EFB) used during the flight test to conduct IM operations. A Configurable Graphics Display (CGD) located in the pilots' primary field of view repeated critical information. The Boeing-757 and Boeing-737 had a Honeywell DO-317A compliant Traffic Processing Unit that provided ADS-B In data to the FIM application hosted on the EFB. The FIM application had a touchscreen interface that enabled the pilots to enter Ownship information, forecast winds, and IM clearance information. The EFB also had a Cockpit Display of Traffic Information (CDTI) that displayed nearby ADS-B Out aircraft. The FIM software and spacing algorithm created by Honeywell were based on NASA ${ }^{23}$ and $\mathrm{RTCA}^{24}$ documents, while the EFB displays ${ }^{25}$ and the $\mathrm{CGD}^{26}$ were influenced by a NASA designed prototype used in previous simulations. ${ }^{27}$

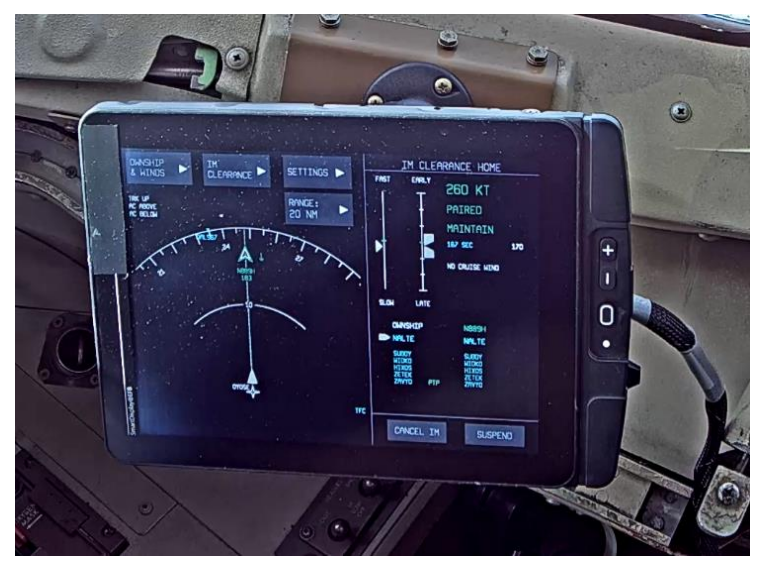

Figure 5. FIM Displays on Electronic Flight Bag. 
There was no data flow from the experimental FIM system to certified avionics.

2. Speed Guidance

The FIM avionics provided the pilots with both speed guidance and speed conformance monitoring. The primary form of speed guidance was a discretized commanded speed displayed using green text on the EFBs and in white text on the CGSs. Pilots were expected to input the discrete commanded speed into their aircraft's mode control panel speed window. When the IM commanded speed changed, the commanded speed was shown in reverse video for 10 seconds. If a new commanded speed was not achieved quickly enough, a speed conformance monitoring function that used the logic described in the MOPS ${ }^{12}$ caused the commanded speed to flash in reverse video.

A secondary form of speed guidance was provided to assist the pilots in modulating the aircraft's thrust and drag during a speed change. This speed guidance was displayed as a fast/slow indicator that depicted the Ownship's speed relative to a reference speed provided by the speed control algorithm. The fast/slow indicator was expected to help obtain consistent spacing performance during large deceleration segments, such as the 210 to 170 knot deceleration on the SUBDY1 arrival and the 240 to 170 knot deceleration on the UPBOB1 arrival (black lines on Figure 2).

\section{Speed Control Algorithm}

To support the four IM clearances, two different speed control laws were implemented in the FIM avionics prototype: a trajectory-based speed control law and a state-based constant time delay speed control law. A general treatment of speed control algorithms for IM operations can be found in references 24,28 , and 29 . The trajectorybased speed control law is used for the achieve-stage of the Cross IM operation (the portion of the operation before the ABP) and the final approach spacing IM operation. The state-based speed control law is used for the maintain phase of the Cross operation (the portion of the operation after the ABP), the Capture operation, and the Maintain operation. These two speed control laws are based largely on version 13 of NASA's ASTAR algorithm. ${ }^{23}$ In general, both control laws calculate a spacing error, a speed correction based on the spacing error, and the speed command as the sum of some nominal speed and the speed correction.

For both speed control laws, kinematic 4-dimensional trajectories for the Ownship and the Target aircraft are produced using a copy of the FMS navigation database hosted in the EFB application along with the sensed wind, and the wind forecast. Since the EFB application does not have knowledge of aircraft dynamics or configuration, the trajectories assume a constant deceleration rate of 0.5 knot per second for descending segments and 0.75 knot per second for level segments. Additionally, a discovery algorithm is used to estimate the Target aircraft's cruise altitude and speed from ADS-B state data.

The spacing error calculated by the trajectory-based speed control law is the difference between the ASG and the predicted spacing interval, which is calculated using trajectory information for the Ownship and Target aircraft. For time-based operations, the predicted spacing interval is the difference in times that the Ownship and Target aircraft are predicted to cross the ABP. For distance-based spacing goals, the predicted spacing interval is the predicted distance between the Ownship and the Target aircraft when the Target aircraft is predicted to cross the ABP. The IM commanded speed is the sum of the Ownship's nominal trajectory speed, a proportional control term to null the spacing error, and a ground speed compensation term that is used to prevent the Ownship from closing too quickly on the Target aircraft.

The spacing error used by the state-based speed control law is the difference between the ASG and the measured spacing interval. Calculating the measured spacing interval relies on historical state data from the Target aircraft, and requires the Ownship to be on the same route as the Target aircraft. For time-based spacing goals, the measured spacing interval is the time elapsed since the Target aircraft crossed the Ownship's current along-path position. For distancebased spacing goals, the measured spacing interval is the along-path distance between the Ownship and Target aircraft. The commanded speed is calculated as the sum of a base speed and a proportional control term. For time-based operations, the base speed is the Target aircraft's time history ground speed that it flew when it was at the Ownship's position, converted to an airspeed. For distance-based operations, the base speed is the Target aircraft's current ground speed converted to an airspeed.

The IM commanded speed produced by the control laws is limited before being displayed to the pilots. Limits to the command speed included the maximum operating speed, a $\pm 15 \%$ boundary around the Ownship's trajectory speed (based on the published procedure), and regulatory speed restrictions. To reduce the number of IM speed changes, hysteresis is applied to the commanded speed, and the speed is discretized to a value of 5 knots when the Ownship is close to the PTP and 10 knots when the Ownship is not close to the PTP.

\section{F. Pilot Training}

In preparation for the flight test, the flight crew and flight test directors received a computer-based course describing the IM concept, equipment, and procedures. After completion, they travelled to NASA Langley Research

American Institute of Aeronautics and Astronautics 
Center to attend a four-day hands-on training course that included classroom training and interactive simulations using the actual arrival and approach procedures. The objectives of the training course and simulations were to familiarize the pilots with the FIM avionics and IM operations and to allow the pilots and flight test director to practice positioning the aircraft to achieve the desired test conditions.

The computer-based training provided a walk-through of the EFB functionality and a description of the flight test airspace. The classroom training and simulation were focused on graduated learning over four days. The first two days focused on the operation of the FIM equipment and the final two days focused on the coordination and crew resource management required to successfully conduct the flight test. Two simulators that emulated the Boeing-737 and Boeing-757 flight test aircraft were connected together and flown with a third pre-recorded aircraft, used as the lead aircraft. Pilots were able to practice manipulating the prototype EFB displays and develop the expertise required to correctly position aircraft prior to the start of each test run. The flight test director was stationed separately from the simulators and was able to interact with the pilots through radio communication, facilitating the establishment of communication protocols used during the flight test.

\section{G. Flight Test Participants}

Flight crews were selected by their respective flight operations department, and included Honeywell flight test pilots and United Airlines flight test and line pilots. All of the pilots were current, qualified to fly the aircraft in the position(s) they flew, had glass cockpit experience, and were RNP qualified. The pilots had 20 to 49 years of flight experience and had between 4,500 to 13,000 hours of total flight time.

\section{Flight Test Results}

The primary focus of the flight test was time-based arrival operations, which aligned with previous fast-time and human-in-the-loop simulations. In addition, descriptive statistics are provided for the distance-based arrival, en route, and final approach operations. Since the FIM avionics contained new software, some software anomalies were discovered during the flight test. Critical anomalies were fixed partway through the flight test, and the data were filtered to remove runs and portions of runs negatively impacted by the anomalies. A goal of less than 10 seconds of spacing error is targeted for IM operations. Some inspection of those outliers which exceeded that operational goal was conducted. The delivery accuracy metrics described below have been independently validated using the ADS-B ground surveillance. The values calculated using the flight test data and the ADS-B ground surveillance data are all well within 0.3 seconds for all time-based clearances.

When interpreting the results, it should be noted that several of the pilots that participated in this flight test were flight test pilots accustomed to flying very precise operation. While all pilots were instructed and trained to fly their aircraft similarly to line pilots, the test pilots often used more speed brake and throttle inputs than would be expected during typical commercial passenger operations.

\section{A. Time-Based Arrival Scenarios}

\section{Maintain Stage Performance}

The maintain stage performance is characterized by two metrics in this paper. First, the maintain stage spacing accuracy indicates how accurately IM aircraft can meet the ASG at the end of the maintain stage. For time-based operations, the maintain stage spacing accuracy is defined as the difference between the ASG and the measured spacing interval at the PTP. Operationally, the maintain stage goal is a spacing error at the PTP within 10 seconds, $95 \%$ of the time. This corresponds to a standard deviation of approximately five seconds if the data are normally distributed. In this flight test, every Maintain, Capture, and Cross-Merge scenario with a maintain stage terminated at ZAVYO, the final approach fix.

Figure 6 and Table 2 show that the arrival scenarios surpassed the performance criteria at the PTP for the Maintain, Capture, and Cross-Merge operations. All time-based Maintain, Capture, and Cross-Merge arrivals had spacing errors within 10 seconds when they crossed ZAVYO. For each operation, the average spacing error was within 2 seconds and the standard deviation was less than 3 seconds. This indicates the ability of those operations to maintain precise spacing to the PTP. These results are consistent with a field evaluation conducted by the United Parcel Service (UPS), MITRE, and the FAA in 2010. They found that all of the aircraft who followed the IM speed commands were able to obtain spacing accuracies within 8 seconds. ${ }^{30}$

American Institute of Aeronautics and Astronautics 


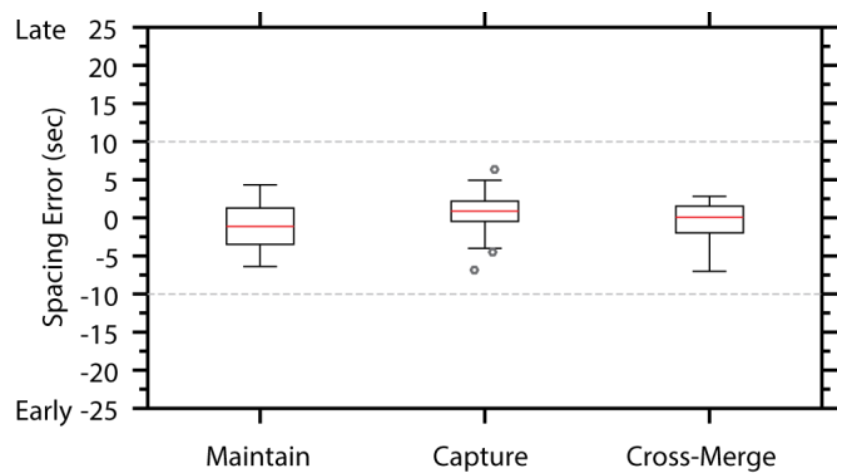

Figure 6: Maintain stage spacing accuracy for timebased arrivals.
Table 2: Maintain stage spacing accuracy for time-based arrivals.

\begin{tabular}{llcc}
\hline $\begin{array}{c}\text { Clearance } \\
\text { Type }\end{array}$ & N & Mean (sec) & SD (sec) \\
\hline Maintain & 18 & -1.13 & 2.99 \\
Capture & 32 & 0.55 & 2.63 \\
Cross-Merge & 27 & -0.47 & 2.45 \\
\hline
\end{tabular}

The second metric comes directly from the FIM standards. Once the spacing error is captured or achieved, the operational goal is to remain within 10 seconds until the PTP. The success criteria described in the FIM MOPS and SPR is to maintain the spacing error within 10 seconds for at least $95 \%$ of the maintain stage operation. For timebased operations, the maintain stage is defined as the entire Maintain operation, the portion of the Capture operation that occurs after the ASG is captured (i.e., after the spacing error is less than 10 seconds), and the portion of the Cross operation after the Ownship crosses the ABP.

Of the 77 operations that contained a maintain phase, 13 did not meet the performance criteria. ${ }^{\ddagger}$ Of these 13 outliers, 7 occurred during the 270 to 210 knot deceleration on the SUBDY1 arrival (black line in Figure 2), and was due to the Ownship and Target aircraft not decelerating at the same rate. Three of the cases were Cross-Merge operations where the spacing error at the ABP was greater than 10 seconds, resulting in spacing errors at the beginning of the maintain stage that were not within 10 seconds. There was one Cross-Merge case where the spacing error was not within 10 seconds due to both the deceleration from 270 knots to 210 knots on the SUBDY1 arrival and a spacing error at the ABP that was not within 10 seconds. The remaining two cases were a Cross-Merge operation that began after the ABP with an initial spacing error greater than 10 seconds, and a Maintain operation where spacing error increased as the Ownship decelerated toward its initial IM commanded speed. Analysis of these outliers reveals that the maintain stage performance was quite good, and that deceleration segments should be considered when designing procedures for use with IM.

\section{Achieve Stage Spacing Accuracy}

The achieve stage spacing accuracy measures how accurately the IM aircraft achieve the ASG at the ABP. For arrival scenarios, this metric only applies to the Cross operations. Similar to the maintain stage spacing accuracy, the achieve stage spacing accuracy is defined as the difference between the ASG and the spacing interval between the Ownship and Target aircraft at the ABP. Again, the operational goal is a spacing error within 10 seconds at the ABP, $95 \%$ of the time. During the arrival scenarios, the ABP was either located at a medium altitude merge point (CrossMerge) or at the FAF (Cross-FAF).

Figure 7 and Table 3 show the spacing performance at the ABP for the Cross-Merge and Cross-FAF operations. The average spacing accuracy of the Cross-FAF operations was -1.65 seconds with a standard deviation of 6.24 seconds. The average spacing accuracy of the Cross-FAF operations was 6.24 seconds with a standard deviation of 8.28 seconds. This performance does not meet the operational goals, though an analysis of the outliers suggest that this is largely attributable to experimental conditions.

Of the 25 Cross-Merge operations, four had spacing errors greater than 10 seconds (16\%). Two of these cases involved conditions at initiation that would not be expected operationally. Both times, the IM operation started within $25 \mathrm{nmi}$ of the ABP, with spacing errors of 19 and 28 seconds early. The Target aircraft had a ground speed 40 knots slower than predicted for the initial 5 to $10 \mathrm{nmi}$ of the operation, likely because it was operating at slower speeds in order to set up the operation. This Target aircraft speed deviation caused the spacing error to increase to a value that was unable to be solved by the ABP, resulting in spacing errors at the ABP of 17 seconds early and 13 seconds early. The other two outliers for Cross-Merge operations appeared to be normal operations with adequate speed control

\footnotetext{
$\ddagger$ One additional case technically did not meet the criteria. For one capture operation, the spacing error was captured when the Ownship was 2.2 nmi prior to the PTP. The small amount of time during the maintain-phase combined with a small amount of noise in the spacing error signal resulted in spacing errors greater than 10 seconds for $6 \%$ of the maintain phase. However, the error was out of conformance for only 3 seconds.
}

American Institute of Aeronautics and Astronautics 


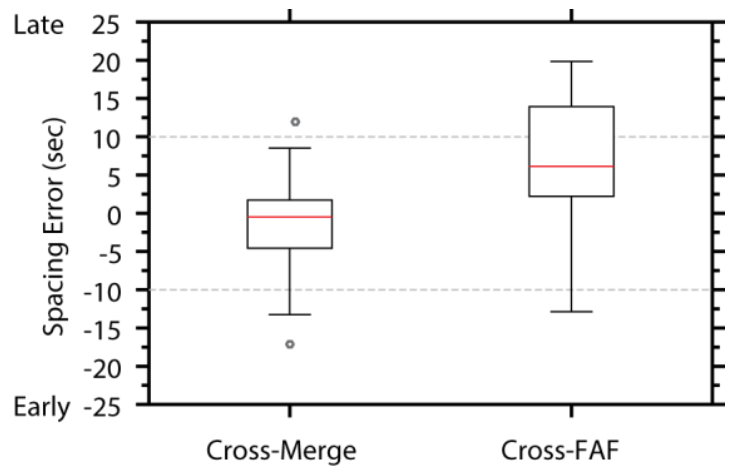

Figure 7: Achieve stage spacing accuracy for timebased arrivals.
Table 3: Achieve stage spacing accuracy for timebased arrivals.

\begin{tabular}{|c|c|c|c|}
\hline Clearance Type & $\mathbf{N}$ & Mean (sec) & SD (sec) \\
\hline Cross-Merge & $25^{*}$ & -1.65 & 6.24 \\
\hline Cross-FAF & 41 & 6.24 & 8.28 \\
\hline
\end{tabular}

authority to null the spacing error. These two cases had spacing accuracies of 12 seconds early and 13 seconds late at the ABP, and will be the subject of future examination.

Of the 41 Cross-FAF operations, 17 had spacing errors at the PTP greater than 10 seconds (41\%). The exact cause of the poor performance of Cross-FAF operations is the subject of further study. Some of the factors that may have contributed to the degraded spacing accuracy are the design of the arrival procedures, speed deviations from the fast/slow indicator guidance, deceleration rates assumed by the spacing algorithm, and differences between the wind forecast and the actual winds.

\section{Spacing Error Capture Rate}

The MOPS defines a minimum capture rate of 3 seconds per minute for the Capture operation. The capture rate was measured as the difference between the initial spacing error and the 10 second threshold, divided by the time it took the Ownship to reduce its spacing error to \pm 10 seconds. Only Capture operations with initial spacing errors greater than 10 seconds were examined.

The data from the time-based Capture operations indicated that only 3 out of the 30 Capture operations did not meet the performance criteria. The first two cases had capture rates of 1.6 and 2.5 seconds per minute. For both of these cases, the Ownship was the last aircraft in the three aircraft string. The lead aircraft (first aircraft in the string) flew a high delay speed profile and the Target aircraft had an early initial spacing error. The Ownship was speed limited by the $\pm 15 \%$ speed bounds applied around the published speeds, indicating that there was not enough speed control authority to capture the ASG at the desired rate. The third case had a capture rate of 2.8 seconds per minute, just missing the design goal. As in the previous two cases, the Ownship was the last aircraft in the string. The lead aircraft (first aircraft in the string) flew a Medium Delay speed profile and the Target aircraft had an early spacing error, limiting the commanded speed to the $\pm 15 \%$ speed bounds during the initial portion of the operation. In all of the other cases, there was sufficient control authority and the prototype FIM avionics met the capture rate criteria.

\section{Speed Command Rate}

The speed command rate indicates how frequently IM speed commands were provided to the pilots. Since the FIM avionics were designed as a retrofit implementation, pilots were required to recognize new speed commands and

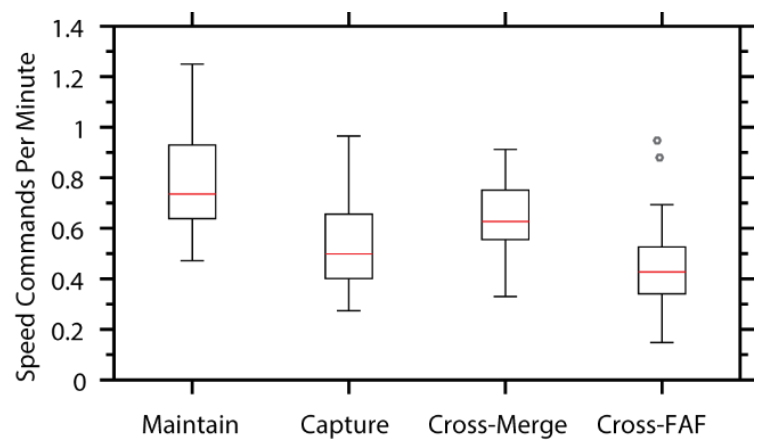

Figure 8: Speed command rate for time-based arrivals.

American Institute of Aeronautics and Astronautics

Table 4: Speed command rate for time-based arrivals.

\begin{tabular}{lccc}
$\begin{array}{c}\text { Clearance } \\
\text { Type }\end{array}$ & $\mathbf{N}$ & $\begin{array}{c}\text { Mean } \\
\text { (number/min) }\end{array}$ & $\begin{array}{c}\text { SD } \\
\text { (number/min) }\end{array}$ \\
\hline Maintain & 18 & 0.80 & 0.23 \\
Capture & 32 & 0.54 & 0.18 \\
Cross-Merge & 27 & 0.64 & 0.15 \\
Cross-FAF & 41 & 0.45 & 0.15 \\
\hline
\end{tabular}


manually input those speeds into their aircraft's mode control panel speed window. Therefore, the speed command rate contributed to pilot task load.

Over all the time-based arrival operations, the average speed command rate was 0.57 speed commands per minute, which equates to approximately one speed command every two minutes. Figure 8 and Table 4 show the speed command rate for each type of IM operation. In general, it appears that the maintain stage experienced more speed commands per minute than the achieve stage. The Cross-FAF operation had the lowest speed command rate, which used the trajectory-based speed control law the entire time, while the Maintain operation had the highest speed command rate and used the state-based speed control law. One potential algorithmic reason why the Maintain operations had a high speed command rate was that both the Target aircraft's time-history ground speed and the spacing error determined the value of the speed command. Therefore, either a change in spacing error or a change in the Target aircraft's ground speed can result in a speed change. It is possible that improved filtering would help eliminate some of the speed changes that were observed.

Data from a survey distributed to the pilots at the end of each operation indicated that that the IM commanded speeds were typically operationally acceptable. The average pilot rating was 5.7 on a Likert scale ranging from "1" (Completely Unacceptable) to "7" (Completely Acceptable). However, pilot comments throughout the flight test indicated that improvements were needed to reduce the number of speed reversals (i.e., cases where there is a speed increase followed by a speed decrease), and the number of instances where several speed changes occur within a short period of time. The behavior of the speed commands will be the subject of further investigation.

\section{B. Distance-Based Arrival Scenarios}

All seven of the distance-based arrival scenarios were Cross operations with the ABP and PTP co-located at the FAF (i.e., Cross-FAF). The initial spacing errors ranged from $2.9 \mathrm{nmi}$ early to $4.1 \mathrm{nmi}$ late and the length of the IM operations ranged from approximately $41 \mathrm{nmi}$ to $99 \mathrm{nmi}$.

The desired spacing performance for distance-based operations is the 10 second time-based tolerance converted to distance using the Ownship's and Target aircraft's ground speeds (approximately $\pm 0.5 \mathrm{nmi}$ for arrival operations). Figure 9 shows that the distance-based CrossFAF operation achieved the desired spacing performance at the PTP for only three out of seven distance-based arrival scenarios.

The two cases that were $1.5 \mathrm{nmi}$ late occurred on a day with strong winds at low altitudes. As the Target aircraft flew around the radius-to-fix turn, the wind it experienced
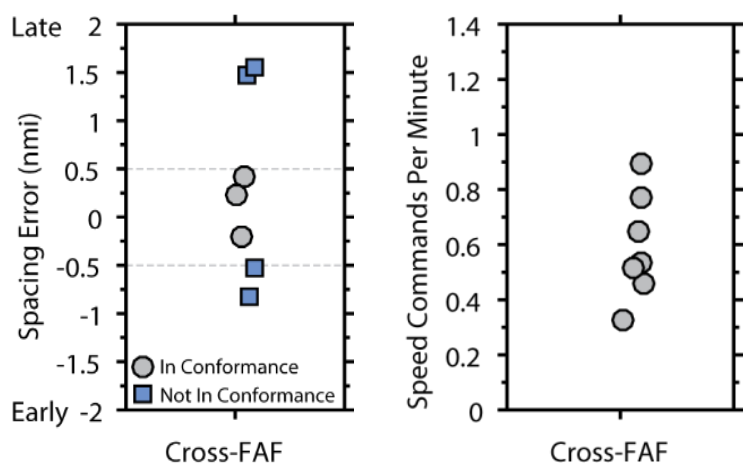

Figure 9. Spacing accuracy at the PTP (left) and speed command rate (right) for distance-based arrivals.

changed from a 60 knot headwind to a 10 knot tailwind. At around the same time, the spacing error began to diverge from zero. A similar increase in spacing error was observed for the time-based Cross-FAF scenarios conducted on the same day; however, the Ownship was able to correct the spacing error after the Target aircraft crossed the PTP. Further examination is required to determine why such a large percentage of the distance-based operations did not achieve the desired spacing accuracy.

The speed command rate shown in Figure 9 has an average of 0.6 speed changes per minute with a standard deviation of 0.2 speed commands per minute, which is similar to the speed command rate observed in the time-based Cross-FAF arrival scenarios. During distance-based operations, the IM performance is met when the Target aircraft crosses the PTP. After the Target aircraft crossed the PTP, there was an average of 3.7 additional speed commands provided to enable the Ownship to match the ground speed of the Target aircraft at the PTP. The speed command rate presented in this paper does not include those additional speed commands. The average pilot rating of the operational acceptability of speeds during distance-based arrival operations was 5.8 on a Likert scale ranging from "1" (Completely Unacceptable) to “7” (Completely Acceptable).

\section{En Route Scenarios}

The eleven en route operations consisted of six time-based operations and five distance-based operations. The length of the time-based en route operations ranged from $49 \mathrm{nmi}$ to $71 \mathrm{nmi}$, and the initial spacing errors of the Capture operations ranged from 22 seconds early to 20 second early. The length of the distance-based en route operations

American Institute of Aeronautics and Astronautics 


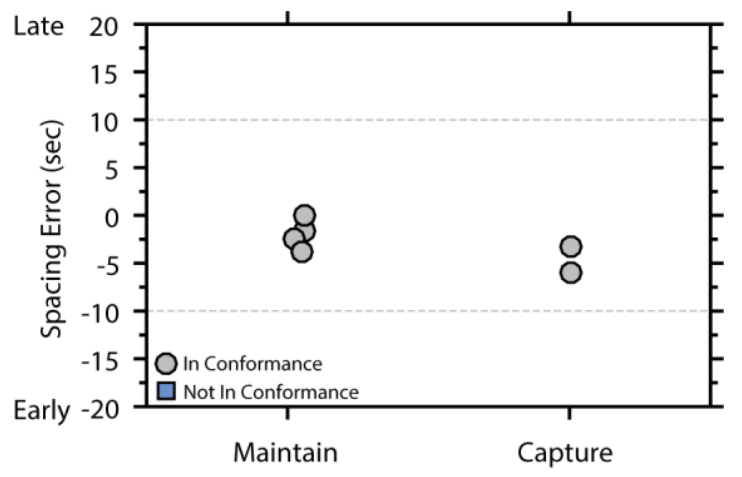

Figure 10. Spacing accuracy at the PTP for timebased en route scenarios.

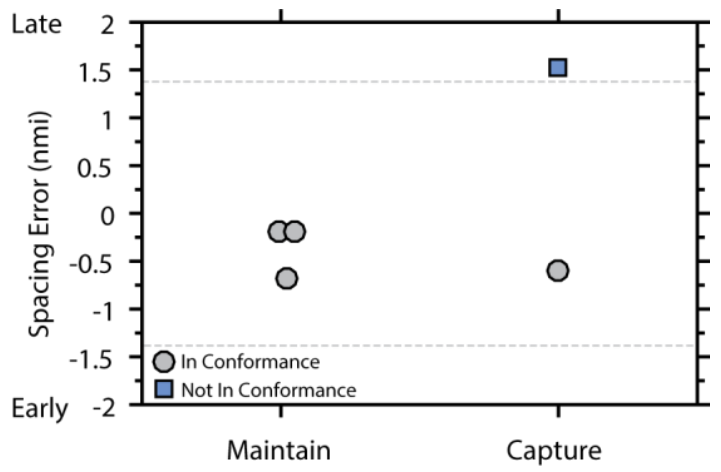

Figure 11. Spacing accuracy at the PTP for distance-based en route scenarios.

ranged from $39 \mathrm{nmi}$ to $67 \mathrm{nmi}$, and the initial spacing errors of the Capture operations ranged from $3.8 \mathrm{nmi}$ early to 2.6 mi late. ${ }^{\S}$ By definition, the initial spacing errors of the maintain operations were close to zero.

Figure 10 shows all of the spacing errors at the PTP for the time-based en route operations were well within the 10 second performance criteria for both the Maintain and Capture operations. The average spacing error at the PTP for time-based en route scenarios was 2.8 seconds early with a standard deviation of 2.1 seconds. Both the maintain stage performance and the capture rate were examined to determine if they met the MOPS criteria. All of the operations maintained the spacing error within 10 seconds at least $95 \%$ of the time throughout the maintain stage. Of the two time-based Capture operations, one did not meet the minimum capture rate of 3 seconds per minute. Further analysis revealed that the capture rate was lower because it took the aircraft some time to decelerate to its initial commanded speed. The average speed command rate was 0.3 speed changes per minute, equivalent to approximately one speed command every three minutes.

Figure 11 shows that four out of five spacing errors at the PTP for the distance-based en route operations were within the distance-based equivalent of the 10 second criteria, which ranged from 1.3 to 1.6 nmi depending on the ground speeds of the aircraft (the grey dashed line in figure 11 shows an approximation of this criteria). On average, the aircraft had a spacing error of $0.03 \mathrm{nmi}$ at the PTP with a standard deviation of $0.9 \mathrm{nmi}$. The one aircraft that did not meet the performance criteria was only $0.1 \mathrm{nmi}$ outside the criteria and in the process of capturing the ASG. Both the maintain stage performance and the Capture rate were examined to determine if they met the MOPS criteria. All four of the distance-based operations that contained a maintain stage were able to maintain the spacing error within 10 seconds, $95 \%$ of the time. The one Capture operation that did not meet the minimum capture rate of 3 seconds per minute was the same operation that that did not achieve a spacing error within 10 seconds at the PTP. The average speed command rate was 0.8 speed commands per minute $(\mathrm{SD}=0.5)$. Similar to the distance-based arrival scenarios, the speed command rate did not include speeds commanded after the Target aircraft crossed the PTP (i.e., after the spacing performance was met).

\section{Final Approach Scenarios}

The eight Final Approach Spacing operations consisted of five time-based operations and three distance-based operations. The length of the time-based Final Approach Spacing operations ranged from $14 \mathrm{nmi}$ to $32 \mathrm{nmi}$, with initial spacing errors ranging from 23 seconds early to 29 seconds late. The length of the distance-based Final Approach Spacing operations ranged from $18 \mathrm{nmi}$ to $22 \mathrm{nmi}$, with initial spacing errors ranged from $1.5 \mathrm{nmi}$ early to $0.1 \mathrm{nmi}$ early.**

Figure 12 shows the spacing error at the PTP for all time-based Final Approach Spacing operations was less than the 10 second performance criteria. The average spacing error was 3.3 seconds with a standard deviation of 4.2 seconds. There was one case that barely achieved the tolerance. Closer examination revealed that the Ownship flew an average of 9.8 knots slower than the desired speed (shown on the fast/slow indicator) throughout the final deceleration, which may have contributed to the spacing error at the PTP. There was an average of 5.2 speed commands per operation, which corresponded to an average speed command rate of 0.9 speed commands per minute with a standard deviation of 0.3 (Figure 13). Data from a survey distributed to the pilots at the end of each operation indicated

\footnotetext{
$\S$ Since the state-based speed control law is used for the Capture and Maintain scenarios, the distance-based initial spacing error is the along path distance between the Ownship and the Target aircraft.

${ }^{* *}$ Since the trajectory-based speed control law is used for final approach spacing operations, the distance-based spacing error is the expected distance error at the PTP if both the IM and Target aircraft were to fly their expected trajectories.
}

American Institute of Aeronautics and Astronautics 

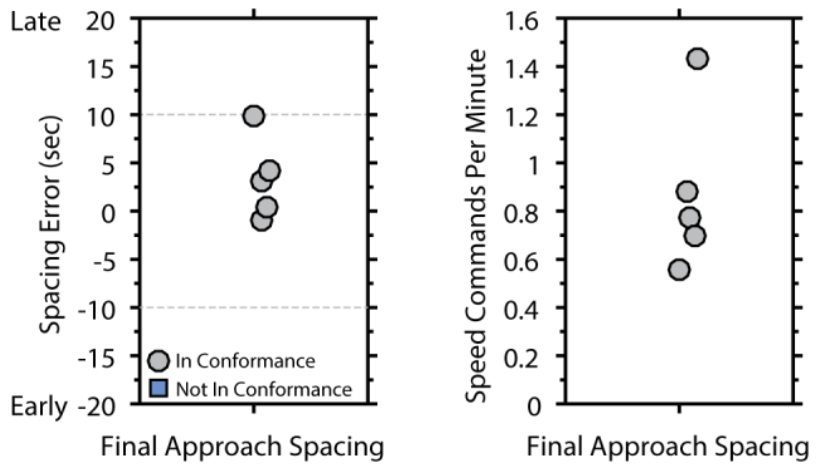

Figure 12. Spacing accuracy at the PTP (left) and speed command rate (right) for time-based final approach scenarios.
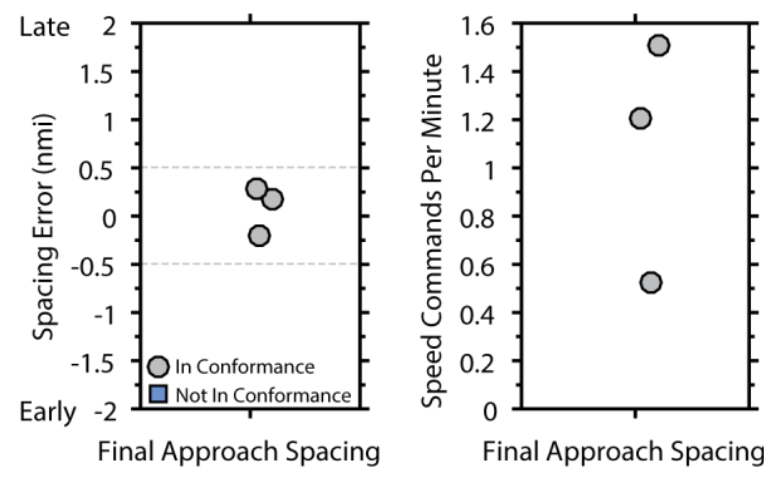

Figure 13. Spacing accuracy at the PTP (left) and speed command rate (right) for distance-based fiı approach scenarios.

that the IM commanded speeds provided were operationally acceptable. All of the pilots rated the acceptability of the commanded speeds as a 6 or higher on a Likert scale ranging from "1" (Completely Unacceptable) to "7" (Completely Acceptable).

Figure 14 shows the spacing error at the PTP for all three distance-based Final Approach Spacing operations were within the desired tolerance, which was 10 seconds converted to a distance (approximately $0.5 \mathrm{nmi}$ ). The average spacing error was $0.09 \mathrm{nmi}$ with a standard deviation of $0.25 \mathrm{nmi}$. There was an average of 3 speed commands per operation, corresponding to an average speed command rate of 1.1 speed commands per minute with a standard deviation of 0.5 (Figure 13). The number of speed commands and speed command rate do not include an average of 1.0 additional speed command provided after the Target aircraft crossed the PTP (after the spacing performance was met). Data from a survey distributed to the pilots at the end of each operation indicated that the IM commanded speeds provided were operationally acceptable. All of the pilots rated the acceptability of the commanded speeds as a 6 or higher on a Likert scale ranging from "1" (Completely Unacceptable) to "7" (Completely Acceptable).

\section{Conclusions}

NASA contracted with Boeing, Honeywell, and United Airlines to build a FIM avionics prototype based on NASA's ASTAR algorithm, and to conduct a flight test which occurred over a period of 19 days in the vicinity of Grant County International Airport. The flight test examined four different types of IM operations in the en route, arrival, and final approach phases of flight. Both the spacing accuracy and the speed command behavior was examined. The results indicated that aircraft were typically able to attain spacing errors less than 10 seconds, or the equivalent distance. However, there were cases where the spacing errors at the PTP and ABP were not within 10 seconds. Most of these cases were Cross arrival scenarios with the ABP and PTP co-located at the FAF (i.e., Cross-FAF). The factors that contributed to the degraded spacing accuracy of the Cross-FAF scenarios will be the subject of future analysis. Inspection of the other outliers across the data set shows many of the other operations which did not meet the 10 second tolerance can be attributed to flight test conditions that were outside of what would be expected operationally.

The rate at which speed commands were provided to the pilots was also examined. For time-based arrivals, the results indicate that the Maintain operation had the highest speed command rate and the Cross-FAF operation had the lowest speed command rate. While the pilots generally rated the speed commands as acceptable, their comments indicated that the acceptability of the speed commands would be greatly improved if there were fewer speed reversals and instances where there are multiple speed changes within a short period of time.

\section{Acknowledgments}

The authors are indebted to many NASA, Boeing, Honeywell, and United Airlines people who went above and beyond what was asked of them to make this flight test successful. Without their contributions the flight test would not have been possible. The Honeywell software team in Redmond, WA and Bangalore, India deserve special recognition for the many hours spent in the lab developing and testing the FIM prototype. The flight test was also assisted by many FAA organizations, including the SBS office, ANG, ANM-220, and five Branches in the ASF-400 Division. The data analysis presented in this paper was also assisted by researchers at the FAA's William J Hughes Technical Center. The professionalism and skill of the controllers, especially Washington Center, was incredible and they have rightfully earned the reputation as the premier place to go for flight testing air traffic management concepts.

American Institute of Aeronautics and Astronautics 
Finally, a special thanks to Cristin Mayes, NASA Intern, who helped write a large portion of the software that was used to analyze the data presented in this paper.

\section{References}

${ }^{1}$ Williams D. H., "Time-based self-spacing techniques using cockpit display of traffic information during approach to landing in a terminal area vectoring environment," NASA-TM-84601, 1983.

${ }^{2}$ Oconnor S., Palmer E., Baty D., and Jago S., "The effect of viewing time, time to encounter, and practice on perception of aircraft separation on a cockpit display of traffic information," A-8072; NASA-TM-81173, Feb. 1980.

${ }^{3}$ Abbott T. S. and Moen G. C., "Effect of Display Size on Utilization of Traffic Situation Display for Self-Spacing Task," NASA-TP-1885, 1981.

${ }^{4}$ Barmore, B., Penhallegon, W. J., Weitz, L. A., Bone, R. S., Levitt, I., Flores Kriegsfeld, J., Arbuckle, D. and Johnson, W.C., 2016. Interval Management: Development and Implementation of an Airborne Spacing Concept. In AIAA Guidance, Navigation, and Control Conference (p. 1608).

${ }^{5}$ Isaacson, D. R., Robinson III, J. E., Swenson, H., and Denery, D., “A Concept for Robust, High Density Terminal Air Traffic Operations," 10th AIAA Aviation Technology, Integration, and Operations (ATIO) Conference, Fort Worth, TX, 2010.

${ }^{6}$ Swenson, H. N., Thipphavong, J., Sadovsky, A., Chen, L., Sullivan, C., and Martin, L., "Design and Evaluation of the Terminal Area Precision Scheduling and Spacing System," Ninth USA/Europe Air Traffic Management Research and Development Seminar (ATM) Berlin, Germany, 2011.

${ }^{7}$ Callantine, T., Palmer, E., and Michael, K., "Human-in-the-loop Simulation of Trajectory-based Terminal-area Operations," $27^{\text {th }}$ International Congress of the Aeronautical Sciences (ICAS), Nice, France, 2010.

${ }^{8}$ Kupfer, M., Callantine, T., Martin, L., Mercer, J., and Palmer, E., "Controller Support Tools for Schedule-based Terminal area Operations," Proceedings of the Ninth USA/Europe Air Traffic Management Research and Development Seminar, 2011.

${ }^{9}$ Robinson III, J. E., Thipphavong, J., and Johnson, W. C., "Enabling Performance-Based Navigation Arrivals," Eleventh USA/Europe Air Traffic Management Research and Development Seminar (ATM2015), Lisbon, Portugal, 2015.

${ }^{10}$ Witzberger, K., Martin, L., "Paradigm Changes Related to TSAS," Journal of Air Traffic Control, Winter 2015, pp. 38-46.

${ }^{11}$ RTCA SC-186, "Safety, Performance, and Interoperability Requirements Document for Airborne Spacing - Flight Deck Interval Management (ASPA-FIM)" DO-328A, Sept 2015.

${ }^{12}$ RTCA SC-186, "Minimum Operational Performance Standards (MOPS) for Flight-deck Interval Management (FIM)," DO361, Sept 2015.

${ }^{13}$ Thipphavong, J., Jung, J., Swenson, H. N., Witzberger, K. E., Lin, M. I., Nguyen, J., Martin, L., Downs, M. B., and Smith, T. A., "Evaluation of the Controller-Managed Spacing Tools, Flight-deck Interval Management and Terminal Area Metering Capabilities for the ATM Technology Demonstration \#1," USA/Europe ATM R\&D Seminar (ATM2013), Chicago, Illinois, 10-13 June 2013.

${ }^{14}$ Callantine, T. J., Kupfer, M., Martin, L., Mercer, J., and Prevot, T., "System-Level Performance Evaluation of ATD-1 Ground-Based Technologies," 14th Aviation Technology, Operations, and Integration Conference, Atlanta, 2014, pp. 16-20.

${ }^{15}$ Wilson, S. R., Kibler, J. L., Hubbs, C. E., and Smail, J. W., "Air Traffic Management Technology Demonstration-1 Research and Procedural Testing of Routes," NASA/TM-2015-218707, May 2015.

${ }^{16}$ Roper, R., "ATD-1 EcoDemonstrator ASTAR Guided Arrival Research (EAGAR)," NASA Briefing, Document ID 20160006919 (available on ntrs.nasa.gov), Jan 2015

${ }^{17}$ Baxley, B., et al., "Human-in-the-Loop Assessment of Alternative Clearances in Interval Management Arrival Operations," NASA/TP-2016-219362.

${ }^{18}$ Baxley, B., Wilson, S., Roper, R., Swieringa, K., "Flight Crew Responses from the Interval Management Alternative Clearances (IMAC) Human-In-The-Loop Experiment," $16^{\text {th }}$ AIAA Aviation Technology, Integration, and Operations Conference (ATIO), AIAA 2016-3299, Washington, D.C., June 2016.

${ }^{19}$ Swieringa, K., Wilson, S., Baxley, B., "System Performance of an Integrated Airborne Spacing Algorithm with Ground Automation," $16^{\text {th }}$ AIAA Aviation Technology, Integration, and Operations Conference (ATIO), AIAA 2016-3900, Washington, D.C., June 2016.

${ }^{20}$ Baxley, B., Palmer, M., Swieringa, K., "Cockpit Interfaces, Displays, and Alerting Messages for the Interval Management Alternative Clearances (IMAC) Experiment,” NASA/TM-2015-218775.

${ }^{21}$ Baxley, B., Swieringa, K., Wilson, S., Roper, R., Hubbs, C., Goess, P., Shay, R., "Flight Crew Survey Responses from the Interval Management (IM) Avionics Phase 2 Flight Test," $17^{\text {th }}$ AIAA Aviation Technology, Integration, and Operations Conference (ATIO), AIAA 2017-2645387, Denver, CO, June 2017.

${ }^{22}$ Brown, J., "NASA ATD-1 Avionics Phase 2 Flight Test Plan,” NASA/CR-2017-219595.

${ }^{23}$ Abbott, T., "An Overview of a Trajectory-Based Solution for En Route and Terminal Area Self-Spacing: Eight Revision," NASA/CR-2017-xxxxxx, submitted for publication.

${ }^{24}$ RTCA SC-186, “Minimum Operational Performance Standards (MOPS) for Flight-deck Interval Management (FIM),” DO361, Sept 2015.

${ }^{25}$ Alves, E., "FIM Avionics Operations Manual,”NASA/CR-2017-219593.

${ }^{26}$ Wilber, G., "ATM Technology Demonstration-1 Phase II Boeing Configurable Graphical Display (CGD) Software Design Description,” NASA/CR-2017-219594.

American Institute of Aeronautics and Astronautics 
${ }^{27}$ Baxley, B., Palmer, M., Swieringa, K., "Cockpit Interfaces, Displays, and Alerting Messages for the Interval Management Alternative Clearances (IMAC) Experiment," NASA/TM-2015-218775.

${ }^{28}$ Weitz, L., and Hurtado, J. String Stability Analysis of Selected Speed Control Laws for Interval Management, AIAA GNC Conference, Aug 2012

${ }^{29}$ Weitz, L., Ganghuai, W., and Stassen, H. Investigating the Performance of Two Interval Management Algorithms in a Fasttime Simulation Environment, AIAA GNC Conference, Aug 2013

${ }^{30}$ Penhallegon, W. J., Bone, R. S., and Stassen, H. (2016). Results from a Field Evaluation of Interval Management during an Optimized Profile Descent Arrival and Approach. In AIAA Guidance, Navigation, and Control Conference (p. 1612). 